\title{
HD 135344B: a young star has reached its rotational limit ${ }^{\star}$
}

\author{
A. Müller ${ }^{1,2}$, M. E. van den Ancker ${ }^{1}$, R. Launhardt ${ }^{2}$, J. U. Pott ${ }^{2}$, D. Fedele ${ }^{3}$, and Th. Henning ${ }^{2}$ \\ ${ }^{1}$ European Southern Observatory, Karl-Schwarzschild-Str. 2, 85748 Garching b. München, Germany \\ 2 Max Planck Institute for Astronomy, Königstuhl 17, 69117 Heidelberg, Germany \\ e-mail: amueller@mpia.de \\ 3 Johns Hopkins University, Dept. of Physics and Astronomy, 3701 San Martin drive, Baltimore, MD 21210, USA
}

Received 16 February 2011 / Accepted 23 March 2011

\begin{abstract}
Aims. We search for periodic variations in the radial velocity of the young Herbig star HD 135344B to determine a rotation period. Methods. We analyze 44 high-resolution optical spectra taken over a time period of 151 days. The spectra were acquired with FEROS at the $2.2 \mathrm{~m} \mathrm{MPG/ESO} \mathrm{telescope} \mathrm{in} \mathrm{La} \mathrm{Silla.} \mathrm{The} \mathrm{stellar} \mathrm{parameters} \mathrm{of} \mathrm{HD} \mathrm{135344B} \mathrm{are} \mathrm{determined} \mathrm{by} \mathrm{fitting} \mathrm{synthetic} \mathrm{spectra} \mathrm{to} \mathrm{the}$ stellar spectrum. To obtain radial velocity measurements, the stellar spectra are cross-correlated with a theoretical template computed from determined stellar parameters.

Results. We report the first direct measurement of the rotation period of a Herbig star from radial velocity measurements. The rotation period is found to be $0.16 \mathrm{~d}(3.9 \mathrm{~h})$, which makes HD 135344B a rapid rotator at or close to its break-up velocity. The rapid rotation could explain some of the properties of the circumstellar environment of HD 135344B such as an inner disk with properties (composition, inclination) that differ significantly from the outer disk.
\end{abstract}

Key words. stars: activity - stars: atmospheres - stars: fundamental parameters - stars: mass-loss - stars: pre-main sequence stars: variables: T Tauri, Herbig Ae/Be

\section{Introduction}

Herbig Ae/Be (HAeBe) stars (Herbig 1960; Finkenzeller \& Mundt 1984; Waters \& Waelkens 1998) are intermediate-mass (2-10 $\left.M_{\odot}\right)$ pre-main-sequence stars. They are believed to be higher-mass counterparts to the T Tauri stars (TTS), hence fill the parameter space between TTSs and high-mass young stars in addressing the question of star formation as a function of mass (Appenzeller 1994).

Stellar rotation is a crucial parameter in the evolution of angular momentum, magnetic fields, and accretion processes. It is known that HAeBe stars exhibit significantly larger projected rotational velocities, $v \sin i$, than TTSs. Typical $v \sin i$ values for $\mathrm{HAeBe}$ stars are in the range from 60 to $225 \mathrm{~km} \mathrm{~s}^{-1}$ (Davis et al. 1983; Finkenzeller 1985; Boehm \& Catala 1995), whereas most TTSs have $v \sin i$ values of about $10 \mathrm{~km} \mathrm{~s}^{-1}$ (e.g. Weise et al. 2010). This result indicates that mechanisms of angular momentum dispersal are much less efficient in HAeBe stars than in TTSs (Boehm \& Catala 1995). Star-disk locking and subsequent rotational braking might even be absent in HAeBe stars. Only for low-mass HAeBe stars $\left(M \leq 2.6 M_{\odot}\right)$ did Boehm \& Catala (1995) find indications of a loss of angular momentum due to stellar winds.

However, no extensive study to determine the rotation periods of $\mathrm{HAeBe}$ stars has yet been presented. Only for a few $\mathrm{HAeBe}$ stars, rotation periods have so far been found. Observed variations of $\mathrm{Ca}$ II $\mathrm{K}$ and $\mathrm{Mg}$ II $\mathrm{h}$ and $\mathrm{k}$ of $\mathrm{AB}$ Aur were interpreted as rotational modulation (e.g. Praderie et al. 1986; Catala et al. 1986). Hubrig et al. (2011) detected a rotationally modulated magnetic field of the HAeBe star HD 101412. In this work,

* Based on observations collected at the European Southern Observatory, La Silla, Chile (Program ID: 084.A-9016, 085.A-9027, 085.A-9024. we determine the rotation period of the Herbig star HD 135344B by measuring radial velocity variations using multi-epoch high resolution optical spectra.

The paper is organized as follows. Section 2 presents the observations and data reduction. The determination of the stellar parameters and the radial velocity measurements are presented in Sects. 3 and 4. A discussion of the results and conclusions can be found in Sects. 5 and 6. The Appendix provides a table of measured quantities.

\section{Observations and data reduction}

The observations were carried out in two observing campaigns with the Fiber-fed Extended Range Optical Spectrograph (FEROS, Kaufer et al. 1999) at the $2.2 \mathrm{~m} \mathrm{MPG/ESO} \mathrm{telescope} \mathrm{at}$ La Silla Observatory in Chile. FEROS covers the whole optical spectral range from $3600 \AA$ to $9200 \AA$ and provides a spectral resolution of $\approx 48000$. The fibre aperture of FEROS is 2 arcsec on the sky. A contamination of the spectra due to other stars around HD 135344B can thus be ruled out (Sect. 3). In total, 44 spectra of HD 135344B were obtained over a time range of five months between March and July 2010. Depending on the conditions, we observed the star up to four times a night with a separation of two hours between the observations. The average exposure time was 16 min per spectrum, which results in an average signal-to-noise ratio $(S N R)$ of 230 at $5500 \AA$ for our spectroscopic data set. A set of 40 spectra were obtained using the object-calibration mode where one of the two fibers is positioned on the target star and the other fiber is fed with the light of a $\mathrm{Th} \mathrm{Ar}+\mathrm{Ne}$ calibration lamp. This mode allows us to monitor and correct for the intrinsic velocity drift of the instrument. An additional four spectra were obtained using the object-sky 
mode where the second fiber points to the sky, allowing the subtraction of the sky background from the target spectrum. The reduction of the raw data was performed using the online data reduction pipeline available at the telescope ${ }^{1}$. The pipeline does the bias subtraction, flat-fielding, traces and extracts the single echelle orders, applies the wavelength calibration, and corrects for the barycentric motion. For each exposure, it produces 39 individual sub-spectra representing the individual echelle orders, as well as one merged spectrum.

\section{Astrophysical parameters of HD 135344B}

HD 135344B (SAO 206462) belongs to the Sco OB2-3 (Upper Centaurus Lupus, UCL) star-forming region, whose center is at a distance of $140 \pm 2$ pc (de Zeeuw et al. 1999). Preibisch \& Mamajek (2008) also list the individual distances of 81 group members. The median and standard deviation of these values indicate that the distance is $142 \pm 27 \mathrm{pc}$. This spread reflects the true extent of Sco OB2-3, rather than observational errors in distances to individual stars. We thus adopt a value of $142 \pm 27 \mathrm{pc}$ for the distance of HD 135344B in the following.

HD 135344B is the secondary star of the visual binary system of HD 135344 (SAO 206463). The two components are separated by $21^{\prime \prime}\left(\mathrm{PA}=197^{\circ}\right.$, Mason et al. 2001), which translates into a projected separation of $3000 \pm 600$ AU for the given distance of $142 \pm 27 \mathrm{pc}$. Therefore, a gravitational interaction between the primary and the disk of HD 135344B can be ruled out for this large separation. Using the HST/NICMOS2 camera at $\lambda=1.6 \mu \mathrm{m}$, Augereau et al. (2001) found a close binary system lying 5.8" from HD 135344B. A companionship was ruled out by the detection of different proper motions by Grady et al. (2009), who used the same instrument.

The disk around HD 135344B has been the subject of numerous studies carried out over a large wavelength range using imaging, spectroscopy, and interferometry (e.g., Thi et al. 2001; Dent et al. 2005; Doucet et al. 2006; Brown et al. 2007; Fedele et al. 2008; Pontoppidan et al. 2008; Grady et al. 2009; Verhoeff et al. 2010) that have identified a complex structure. An outer radius of $200 \mathrm{AU}$ for the dusty disk was derived by Doucet et al. (2006) at $20.5 \mu \mathrm{m}$. By modeling the Spitzer spectrum, Brown et al. (2007) found a gap in the dusty disk between $0.45 \mathrm{AU}$ and 45 AU. We should note that Brown et al. (2007) do not resolve the inner disk but estimate the location of the inner rim of the gap based on potentially ambiguous SED model fits. This agrees with the mid-IR observations of Verhoeff et al. (2010). One explanation of a gap in the disk, besides grain growth and photo-evaporation, is that a (sub-)stellar companion has formed causing a dynamical clearing. Pontoppidan et al. (2008) rule out the presence of a stellar companion around HD 135344B based on their detection of molecular $\mathrm{CO}$ gas $(\lambda=4.7 \mu \mathrm{m})$ at $0.3-$ $15 \mathrm{AU}$.

The value of the disk inclination derived from different observations at different wavelengths varies from $11^{\circ}$ to $61^{\circ}$ (Dent et al. 2005; Doucet et al. 2006; Fedele et al. 2008; Pontoppidan et al. 2008; Grady et al. 2009). We note, however, that different methods probe different spatial scales in the disk. Therefore, these different values may not contradict each other but may reflect the presence of a more complex morphology than assumed in the simple symmetric models from which these values are derived. Grady et al. (2009) ruled out inclinations greater than $20^{\circ}$ for the outer disk, which is in good agreement with the value of

\footnotetext{
1 http://www.eso.org/sci/facilities/lasilla/ instruments/feros/
}

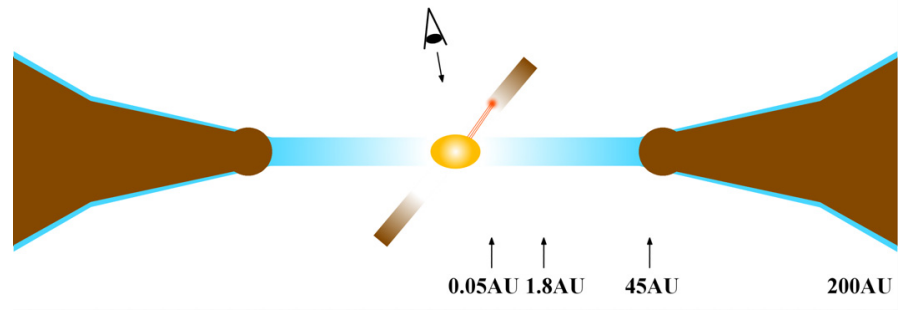

Fig. 1. A pictographic sketch of the HD 135344B system. The star, the dusty disk (brown color) with its large inner gap, the gaseous disk (blue), and an hot spot emerging from the inner rim of the inner disk caused by active accretion (red) are shown.

$11^{\circ} \pm 2^{\circ}$ measured by Dent et al. (2005). Therefore, the outer disk is seen almost face-on as it is already indicated by direct imaging (Doucet et al. 2006; Grady et al. 2009). Interferometric $N$-band observations of the inner part of the star-disk system identified a much higher inclined $\left(53^{\circ}-61^{\circ}\right)$ structure with an estimated inner radius of about $0.05 \mathrm{AU}$ and an outer radius of $1.8 \mathrm{AU}$ (Fedele et al. 2008).

The disk of HD 135344B contains $(2.8 \pm 1.3) \times 10^{-3} M_{\odot}$ of gas and dust (Thi et al. 2001). The detection of polycyclic aromatic hydrocarbon (PAH) features (e.g. Coulson \& Walther 1995; Brown et al. 2007; Fedele et al. 2008) implies that there is rich chemistry in the vicinity of the star. From $J=3-2{ }^{12} \mathrm{CO}$ measurements, Dent et al. (2005) derived an inner radius of $\leq 10 \mathrm{AU}$ and $75 \pm 5 \mathrm{AU}$ for the outer radius where emitting gas is present.

HD 135344B also displays signs of active mass accretion. This matches the findings of Pott et al. (2010), who studied young transitional disk systems with a gap in the circumstellar dust distribution, similar to HD 135344B (Fig. 1). They systematically found sub-AU scale dust in accreting transitional disk systems. Garcia Lopez et al. (2006) derived a value for the mass accretion rate of $\dot{M}_{\text {acc }} \approx 5.4 \times 10^{-9} M_{\odot} \mathrm{yr}^{-1}$ using the $\mathrm{Br} \gamma$ emission line in the NIR. From FUV data, Grady et al. (2009) concluded that HD 135344B drives no jet, but found indications of a stellar wind leading to a mass-loss rate between $10^{-11}$ and $10^{-8} M_{\odot} \mathrm{yr}^{-1}$.

On the basis of all previous observations, the disk can be assumed to contain dust within an inner ring from $0.05 \mathrm{AU}$ to 1.8 AU. This structure is highly inclined relative to the outer disk. There is evidence of mass accretion from the inner disk onto the star. Between 1.8 AU and $45 \mathrm{AU}$, a large gap is present. At 45 AU, the outer disk, seen almost pole-on, begins extending to about $200 \mathrm{AU}$. We refer to Fig. 1 for a pictographic sketch.

\subsection{Stellar parameters}

Table 1 lists the derived stellar parameters of HD 135344B. The luminosity, $L_{\star}$, was computed by integrating the total flux under the SED. The parameters $T_{\text {eff }}, \log g$, and $v \sin i_{\star}$ were computed using a self-developed tool for retrieving stellar parameters of Herbig stars based on fitting synthetic spectra to the observed stellar spectrum. The computation of the synthetic spectrum was carried out using SPECTRUM ${ }^{2}$ (Gray \& Corbally 1994) with the ATLAS9 atmosphere models (Castelli \& Kurucz 2004). For a detailed description of this procedure, we refer to Müller et al. (in prep.). Figure 2 shows a part of a stellar spectrum of HD 135344B (black line, observed at JD $=2455347.63521$ ) that is well fitted by a single star synthetic spectrum (red line).

${ }^{2}$ http://www.phys.appstate.edu/spectrum/spectrum.html 

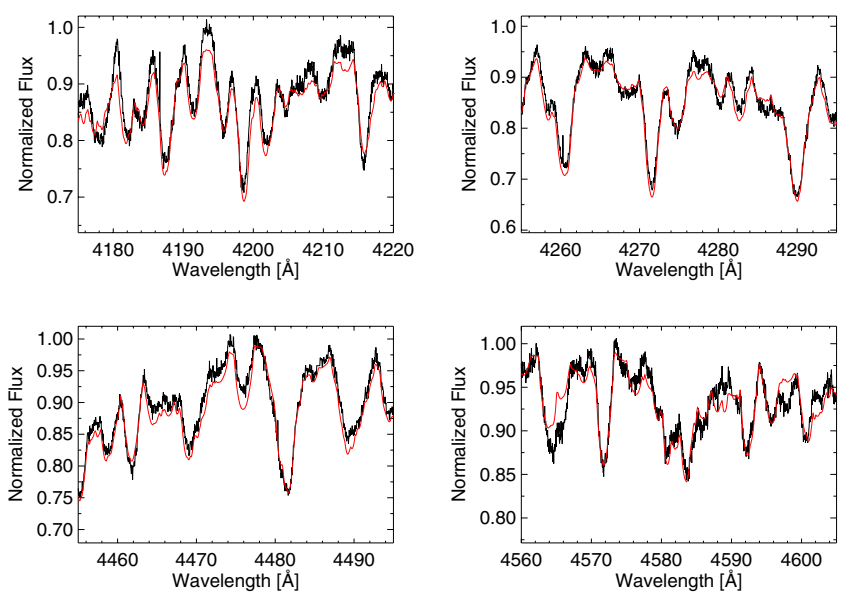

Fig. 2. The spectral windows used for fitting the synthetic spectrum (red line) to the observed stellar spectrum (black line, JD $=2455347.63521$ ) of HD 135344B.

Table 1. Stellar parameters of HD 135344B.

\begin{tabular}{lc}
\hline \hline Parameter & Value \\
\hline distance & $142 \pm 27 \mathrm{pc}$ \\
$\log L_{\star}$ & $1.02_{-0.10}^{+0.18} L_{\odot}$ \\
$T_{\text {eff }}$ & $6810 \pm 80 \mathrm{~K}$ \\
$\log g$ & $4.4 \pm 0.1 \mathrm{~cm} \mathrm{~s}^{-2}$ \\
$M_{\star}$ & $1.7_{-0.1}^{+0.2} M_{\odot}$ \\
age & $9 \pm 2 \mathrm{Myr}$ \\
$R_{\star}$ & $1.4 \pm 0.25 R_{\odot}$ \\
$\left\langle R V_{\star}\right\rangle$ & $2.5 \pm 1.5 \mathrm{~km} \mathrm{~s}^{-1}$ \\
$v \sin i_{\star}$ & $82.5 \pm 2.9 \mathrm{~km} \mathrm{~s}^{-1}$ \\
\hline
\end{tabular}

Notes. $\left\langle R V_{\star}\right\rangle$ represents the mean value of the observed $R V \mathrm{~s}$. The error does not present the accuracy of the determined $R V_{\star}$ but reflects the scatter in $R V_{\star}$ around the mean value.

From the position of HD 135344B in the H-R diagram (Fig. 3), we derived its stellar mass, radius, and age. The derived parameters are in close agreement with literature values, e.g., van der Plas et al. (2008), Manoj et al. (2006), van Boekel et al. (2005), and Dunkin et al. (1997). The measured effective temperature is comparable to that of an F3Ve to F4Ve main-sequence star and agrees with the value found by Dunkin et al. (1997).

\section{Data analysis and results}

\subsection{Radial velocity measurements}

We measured the stellar $R V$ by cross-correlating the stellar spectrum with a template spectrum. The template was a synthetic spectrum representing the fitted stellar parameters of HD 135344B (Table 1). We measured a projected rotational velocity, $v \sin i_{\star}$, of $82.5 \mathrm{~km} \mathrm{~s}^{-1}$ (Sect. 3.1). Therefore, all stellar spectral lines are highly broadened and blended, which makes a determination of $R V_{\star}$ difficult because the accuracy is limited to some $100 \mathrm{~m} \mathrm{~s}^{-1}$. For the cross-correlation, only the spectral range between $4000 \AA$ to $7875 \AA$ was considered because the instrument efficiency drops significantly outside this spectral range. In addition, areas with strong emission lines, telluric lines, and Balmer lines were carefully excluded. The resulting cross-correlation function was fitted by a Gaussian function. The position of the center of the Gaussian yields $R V_{\star}$. After the individual $R V_{\star}$ values were derived separately for each echelle order, the median value and standard deviation of $R V_{\star}$ were

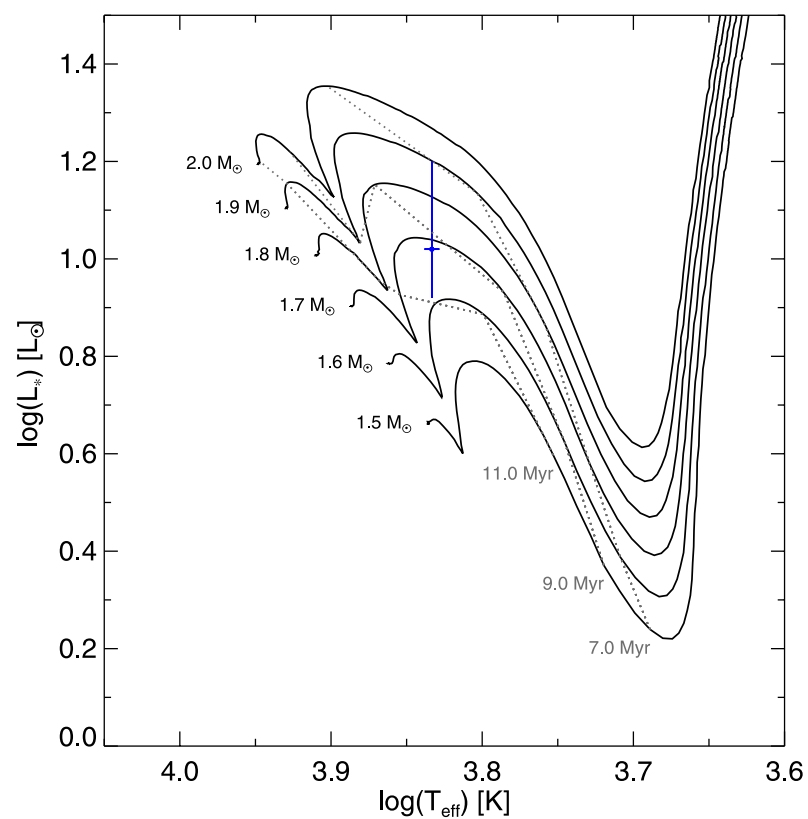

Fig. 3. The position of HD 135344B in an H-R diagram along with the evolutionary tracks of Siess et al. (2000) for a metal abundance of $Z=$ 0.02 .

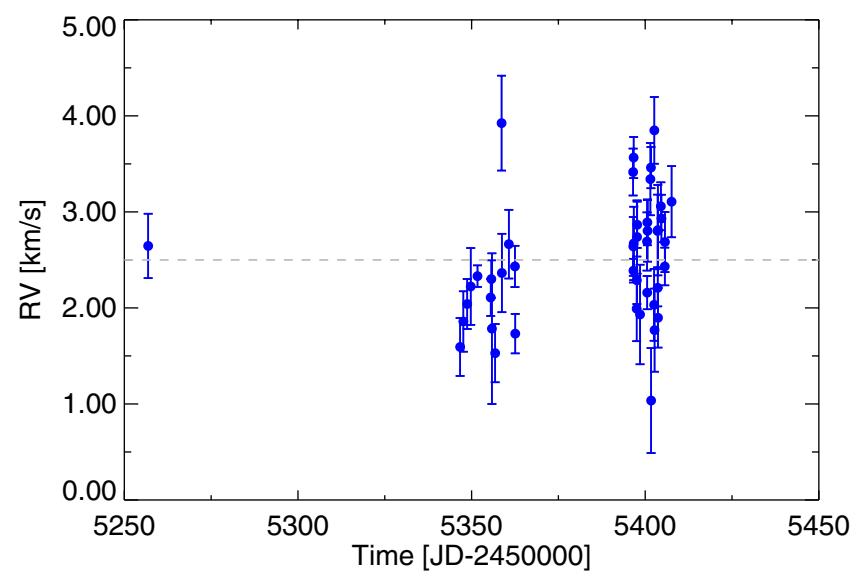

Fig. 4. Measured $R V$ s for HD 135344B. The horizontal gray dashed line represents the mean value of the measurements.

computed. We applied to the $R V_{\star}$ values a $1-\sigma$ clipping to remove anomalous velocity values, similar to e.g. Barnes et al. (2005) and Jeffery et al. (2007). The deficient $R V_{\star}$ estimates, rejected by this $\sigma$ clipping, do not follow a simple Gaussian noise statistics but are far from the median $R V_{\star}$, which indicates that there are individual, systematic biases in the $R V_{\star}$ estimate in the rejected orders. The final $R V_{\star}$ were then computed based on averaging ten to twelve remaining individual orders. The error in the final $R V_{\star}$ is the standard deviation of the mean. All measured $R V_{\star}$ values are listed in Table A.1 provided in the Appendix. Figure 4 shows the measured $R V$ s for all 44 spectra with a mean $R V_{\star}$ value of $2.5 \mathrm{~km} \mathrm{~s}^{-1}$, indicated by the gray horizontal dashed line. Peak-to-peak variations of up to $2.9 \mathrm{~km} \mathrm{~s}^{-1}$ are present.

\subsection{Radial velocity variations}

To identify periodicities present in the $R V_{\star}$ data, we computed the generalized Lomb-Scargle (GLS) periodogram (Zechmeister $\&$ Kürster 2009) and its window function (Fig. 5). The GLS exhibits a strong peak at a period of $0.16045 \mathrm{~d}$ (in the following, we use $0.16 \mathrm{~d})$, marked by the red arrow. Its false-alarm probability 


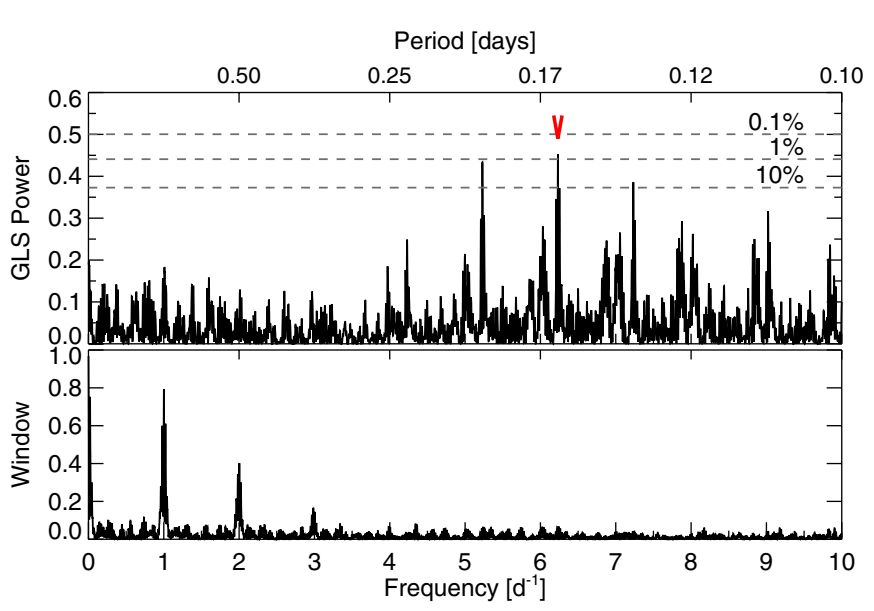

Fig. 5. GLS periodogram (upper plot) and window function (lower plot) of the $R V_{\star}$ data. The significant period at $0.16 \mathrm{~d}$ is marked by the red arrow. FAP thresholds for $0.1 \%, 1 \%$, and $10 \%$ are indicated by the horizontal dashed lines. The data are plotted over frequency but the upper $x$-axis gives the corresponding period. Note that periods greater than $1 \mathrm{~d}$ are in the frequency range between 0 and $1 \mathrm{~d}^{-1}$.

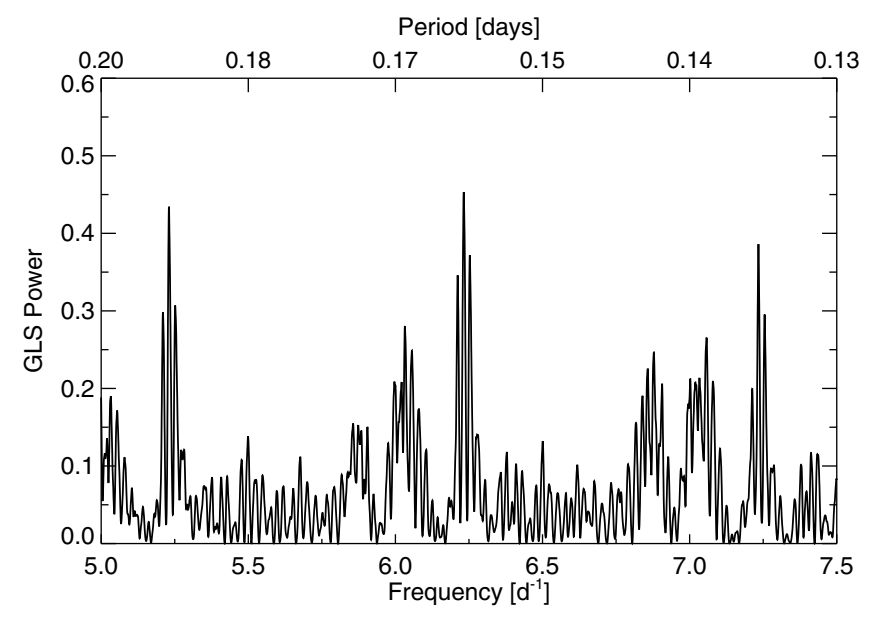

Fig. 6. Close-up view of the GLS centered around the most significant peak at $0.16 \mathrm{~d}$. Two less significant peaks at $5.230 \mathrm{~d}^{-1}$ and $7.234 \mathrm{~d}^{-1}$ are present in addition and are aliases caused by the sidereal day. All three peaks show side lobes with a difference of $0.021 \mathrm{~d}^{-1}$ in frequency, caused by the uneven sampling of our data.

(FAP) is $0.64 \%$. In addition, several other peaks in the GLS are present that are less or not significant compared to the $0.16 \mathrm{~d}$ period. This complicates the interpretation of a periodogram. To identify alias frequencies caused by uneven sampled data in time, we have to consider the window function. Significant peaks in the window function can produce aliases in the GLS that are separated from the true peak by the frequency difference. Figure 6 shows a close-up view of the GLS, centered on the $0.16 \mathrm{~d}$ period (or at the frequency of $6.232 \mathrm{~d}^{-1}$, respectively). The two strongest other but less significant peaks are located at $5.230 \mathrm{~d}^{-1}$ and $7.234 \mathrm{~d}^{-1}$. The difference of both frequencies with respect to $6.232 \mathrm{~d}^{-1}$ is $1.002 \mathrm{~d}^{-1}$, which corresponds to the sidereal day. Figure $7 \mathrm{~b}$ shows a close-up view of the window function centered around the significant frequency $1.0 \mathrm{~d}^{-1}$. The highest peak is at a frequency of $1.002 \mathrm{~d}^{-1}$. We can therefore conclude that the peaks to both the left and right of the $0.16 \mathrm{~d}$ period in the GLS are aliases. Each of the three peaks in Fig. 6 display side lobes separated by $0.021 \mathrm{~d}^{-1}$ for all cases. From the
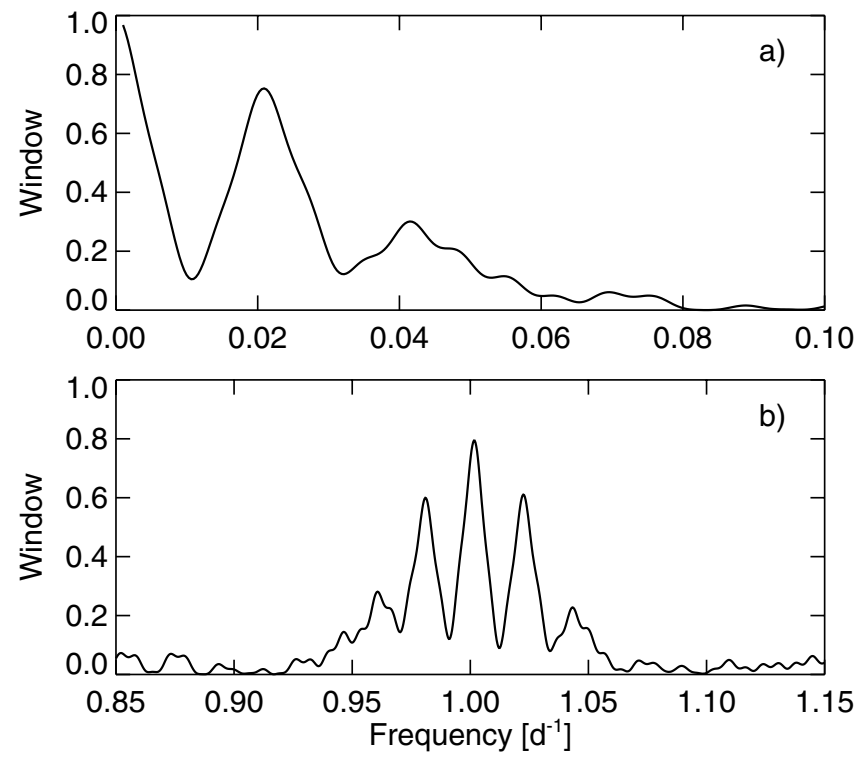

Fig. 7. Close-up views of the window function for the significant peaks at $<0.006 \mathrm{~d}^{-1}, 0.021 \mathrm{~d}^{-1}$, and $1.002 \mathrm{~d}^{-1}$.

Table 2. Results of the sinusoidal fit to the $R V_{\star}$ data.

\begin{tabular}{lc}
\hline \hline Parameter & Value \\
\hline$\chi_{\text {red }}^{2}$ & 2.5 \\
$r m s$ & $478 \mathrm{~m} \mathrm{~s}^{-1}$ \\
period & $0.16045 \pm 2 \times 10^{-5} \mathrm{~d}$ \\
amplitude & $493 \pm 86 \mathrm{~m} \mathrm{~s}^{-1}$ \\
phase (to $\mathrm{JD}_{\min }$ ) & $0.95 \pm 0.03$ \\
offset & $2508 \pm 61 \mathrm{~m} \mathrm{~s}^{-1}$ \\
\hline
\end{tabular}

close-up view of the window function (Fig. 7a), we can identify a significant frequency at $0.021 \mathrm{~d}^{-1}$, which is exactly the measured frequency difference between the side lobes of three main peaks. The $0.021 \mathrm{~d}^{-1}$ frequency corresponds to a period of about 48 d. By looking at the distribution of observations in Fig. 4, we can identify three separate data sets (the first data set consisting of the first single data point only). The time difference between the first and the second data set is about $100 \mathrm{~d}$, and the difference between the second and the third data set is about $50 \mathrm{~d}$. This explains the observed aliases at this frequency difference in the GLS and the presence of a significant peak in the window function. The observations cover a time range of about $150 \mathrm{~d}$. Therefore, the window function (Fig. 7a) shows a significant peak for frequencies smaller than $0.006 \mathrm{~d}^{-1}$ (or periods greater than $150 \mathrm{~d}$ respectively), but they are not visible as side lobes of the main peaks in the GLS. In addition, we subtracted a sinusoidal fit from the $R V_{\star}$ data and computed a GLS periodogram of the residuals (Fig. 9) to verify that the $0.16 \mathrm{~d}$ period is present in the $R V_{\star}$ data. The GLS periodogram of the residuals clearly shows that the peak at $0.16 \mathrm{~d}$ as well as its corresponding aliases was removed. There are no additional significant peaks at other periods. From this analysis, we assume that the $0.16 \mathrm{~d}$ period is significant and indeed the only real period in the observed $R V_{\star}$ data.

Figure 8 shows the phase-folded $R V_{\star}$ data for the $0.16 \mathrm{~d}$ period. The solid line represents a sinusoidal fit to the data with a period of $0.16045 \pm 2 \times 10^{-5} \mathrm{~d}$ and an amplitude of $493 \pm 86 \mathrm{~m} \mathrm{~s}^{-1}$. The results of the fit are presented in Table 2 . 


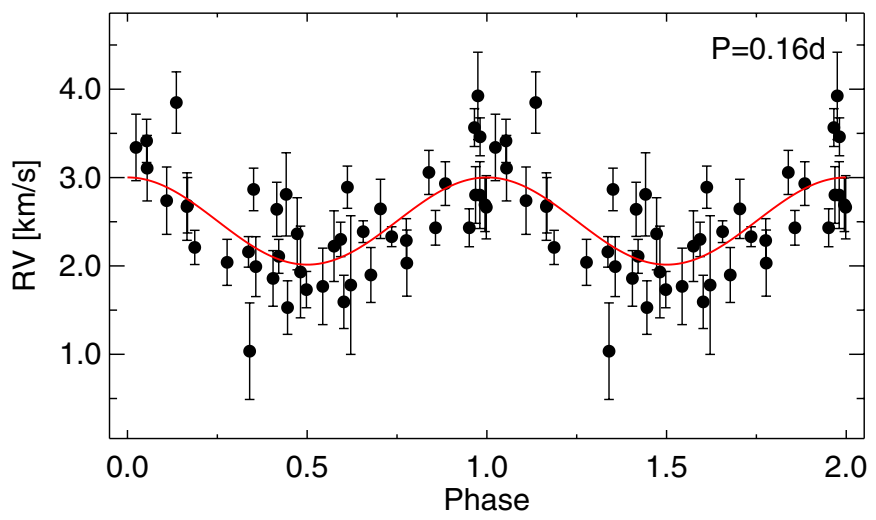

Fig. 8. Phase-folded plot of the $R V_{\star}$ data. The solid line is a sinusoidal fit with a period of $0.16045 \pm 2 \times 10^{-5} \mathrm{~d}$ and an amplitude of $493 \pm$ $86 \mathrm{~m} \mathrm{~s}^{-1}$.

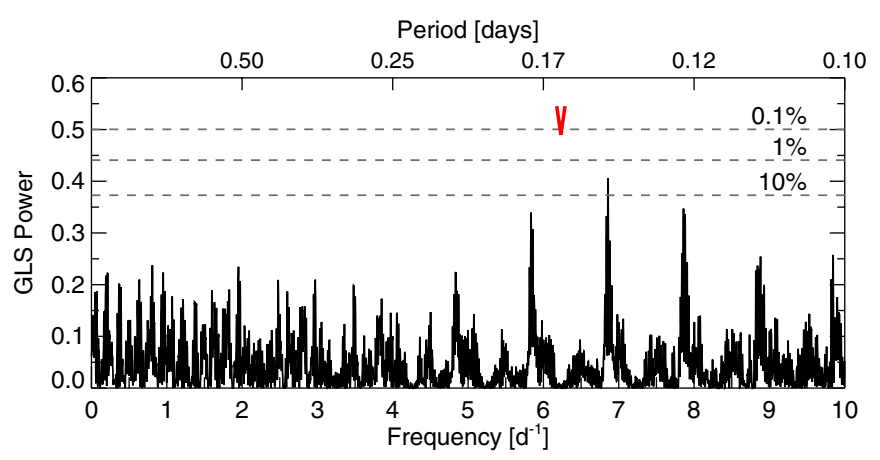

Fig. 9. GLS periodogram of the residual $R V_{\star}$ data after subtraction of the sinusoidal fit. The red arrow marks the position of the $0.16 \mathrm{~d}$ period, which is no longer present, as well as its corresponding aliases. The new peak with the highest power at $0.14 \mathrm{~d}$ has a FAP of $3.4 \%$ and is therefore insignificant.

\subsection{Bisector analysis}

Stellar activity such as cold and hot spots, granulation, and pulsation can mimic significant $R V$ variations that can be periodic because of stellar rotation. For a cold (i.e. dark) spot, the line profile becomes asymmetric because of the fainter stellar light. Stellar rotation leads to a modulation of the asymmetry and causes $R V$ variations depending on the stellar inclination and latitude of the spot. The analysis of the line profile (in the following bisector) is routinely applied to search for extrasolar planets when the $R V$ technique is used to determine whether the observed periodic $R V$ variations are caused by stellar activity or a companion (e.g. Queloz et al. 2001). A correlation between $R V_{\star}$ and bisector velocity span would indicate that the $R V_{\star}$ variation is caused by line shape asymmetries, i.e. by rotational modulation caused, for example, by a spot. The shape of the computed cross-correlation function $(\mathrm{CCF})$ represents the mean line profile of the selected lines in the observed spectra. We measured the bisector velocity span (BVS), bisector displacement (BVD), and bisector curvature (BC) for all our spectra following the definition of Povich et al. (2001). To construct the bisector, the middle points of the horizontal segments, which connect a point on the left to the right side of the CCF at the same flux level, are computed. The bisector is divided into three zones. The BVS is simply the difference between the upper and lower zones. The BC is defined as the difference between the upper and lower bisector spans. To derive the BVD, we calculate the average of the three bisector velocity zones. To compute the individual quantities, we chose heights of $30 \%, 50 \%$, and $85 \%$ of the bisector. As for the

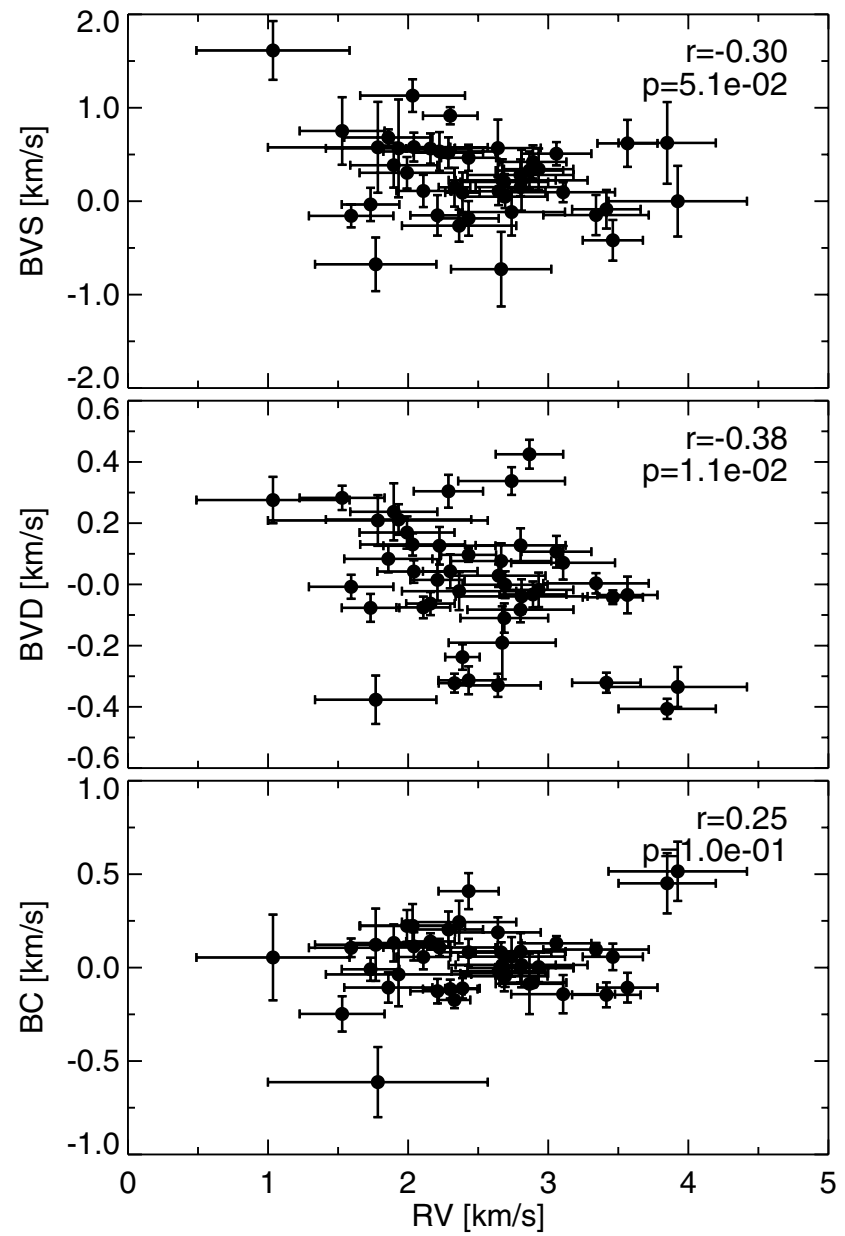

Fig. 10. Bisector quantities versus $R V_{\star}$. For each plot, the linear correlation coefficient $r$ and the probability $p$ that the data are linearly uncorrelated are computed (Bevington \& Robinson 2003). There is no linear correlation between the bisector quantities and $R V_{\star}$.

$R V_{\star}$ measurements, we measured $\mathrm{BVS}, \mathrm{BVD}$, and $\mathrm{BC}$ for each computed $\mathrm{CCF}$. The final bisector values were derived by averaging the single values. The errors in BVS, BVD, and BC are the standard deviations of the means. In Fig. 10, we have plotted all three bisector quantities against $R V$. The computed linear correlation coefficients $r$ are noted in the upper right corner of each plot. We find that there is a significant linear correlation for none of the three quantities. Only a weak trend might be present for BVS and BD. However, the sensitivity of the bisector method decreases rapidly when it comes to low stellar inclinations (e.g. Desort et al. 2007). For HD 135344B, we can assume that we see the star almost pole-on (Sect. 5), hence we do not expect there to be a strong correlation between $\mathrm{BVS}$ and $R V$.

\section{4. $\mathrm{H} \alpha$ measurements}

The $\mathrm{H} \alpha$ line profile of HD 135344B undergoes rapid and significant changes. Finkenzeller \& Mundt (1984) classified the $\mathrm{H} \alpha$ line profile of $\mathrm{HAeBe}$ stars into three categories: singlepeak, double-peak, and P Cygni profiles. The $\mathrm{H} \alpha$ line profile of HD 135344B adopt each of these types at different times. Figure 11 shows the $\mathrm{H} \alpha$ line profiles of our spectroscopic data set of HD 135344B ordered with respect to their phase value according to a $5.77 \mathrm{~d}$ period. A double-peaked profile corresponds 


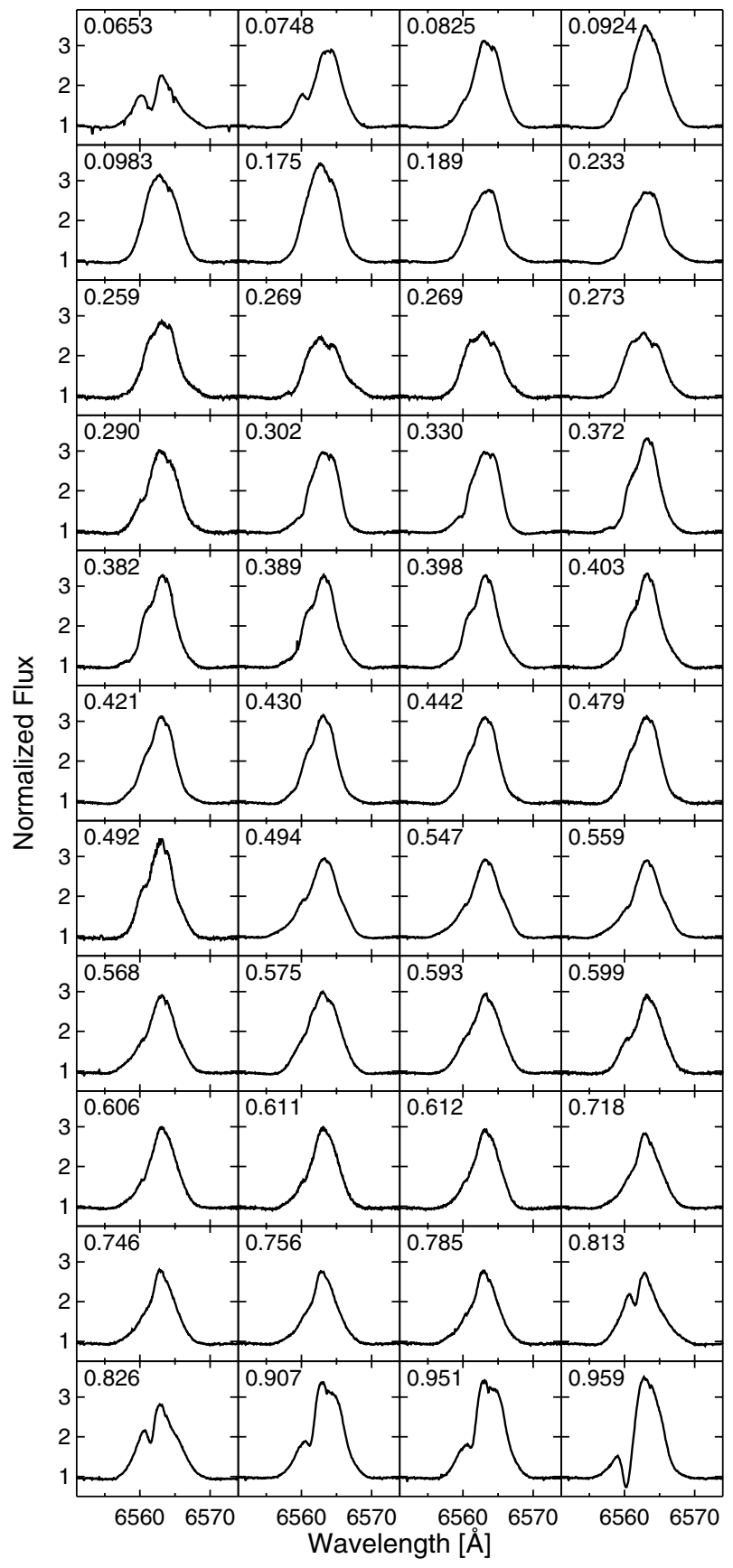

Fig. 11. $\mathrm{H} \alpha$ line profiles of HD 135344B. The plots are ordered with respect to their phase value according to a $5.77 \mathrm{~d}$ period, which is shown in the upper left corner of each plot.

to accretion, whereas a blue-shifted absorption (P Cygni profile) represents an outflow of material. We measured the equivalent width, $E W(\mathrm{H} \alpha)$, of HD 135344B in our FEROS spectra. The measured values are listed in Table A.1 in the Appendix. To measure $E W(\mathrm{H} \alpha)$, we selected several distinct wavelength intervals on both sides of the $\mathrm{H} \alpha$ line and used a second order polynomial to describe the continuum. This was repeated four times for different intervals. The deviation of all four measurements defines our error. For $E W(\mathrm{H} \alpha)$, we obtained a mean value of $-10 \AA$. We were unable to reliably determine a period from the GLS periodogram of the $E W(\mathrm{H} \alpha)$ values.

We also measured the full width of $\mathrm{H} \alpha$ at $10 \%$ height, $W_{10}(\mathrm{H} \alpha)$, which is frequently used for TTSs to distinguish

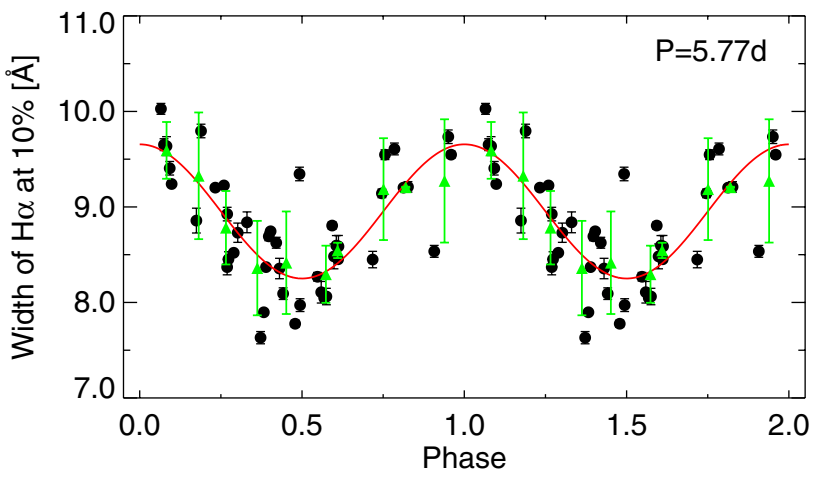

Fig. 12. Phase-folded plot of the $W_{10}(\mathrm{H} \alpha)$ data. The solid line is a sinusoidal fit with a period of $5.77 \pm 0.02 \mathrm{~d}$ and an amplitude of $0.70 \pm 0.08 \AA$. The green triangles are the average values computed by binning the phase in 0.1 wide bins. Their error bars are derived by computing the standard deviation of the corresponding $W_{10}(\mathrm{H} \alpha)$ values in each bin.

between chromospheric activity and accretion of circumstellar material (White \& Basri 2003; Natta et al. 2004; Jayawardhana et al. 2006). We applied the same method to normalize the spectra as for $E W(\mathrm{H} \alpha)$. We measured a mean value of $9.8 \AA$ for $W_{10}(\mathrm{H} \alpha)$, which corresponds to an accretion rate of $3 \times$ $10^{-9} M_{\odot} \mathrm{yr}^{-1}$ if Eq. (5) in Natta et al. (2004) is applied. The measured values of $W_{10}(\mathrm{H} \alpha)$ are listed in Table A.1 provided in the Appendix. The periodogram of $W_{10}(\mathrm{H} \alpha)$ has a significant period at $5.77 \mathrm{~d}$ with a FAP of $1.9 \times 10^{-6}$. A phase-folded plot for the $W_{10}(\mathrm{H} \alpha)$ data (black data points) and the sinusoidal fit (red line) is shown in Fig. 12. Assuming Keplerian rotation of the circumstellar gas, this period corresponds to a radial distance of $\approx 0.07 \mathrm{AU}$. The error bars of $W_{10}(\mathrm{H} \alpha)$ are the statistical errors in the single measurements and reflect the precision of the individual measurements. These error bars most likely overestimate the accuracy of each measurement, since the distance of the data points to the sinusoidal fit often exceeds the error bars significantly. A possible reason for an accuracy bias in the individual $W_{10}(\mathrm{H} \alpha)$ measurements is that the $\mathrm{H} \alpha$ line can form at different locations. The formation might also be caused by different processes, e.g. accretion column, accretion shock on the stellar surface, disk surface, and wind. Therefore, we see a significant scatter in the measured $W_{10}(\mathrm{H} \alpha)$ values around the sinusoidal fit, the level of which exceeds the precision of the individual measurements. However, the bias in each individual measurement is statistically spread around the fit. We binned the phase in 0.1 wide bins and averaged the $W_{10}(\mathrm{H} \alpha)$ values lying in the corresponding bin. The displayed error is the standard deviation of the values in each bin. These values are overplotted in Fig. 12 with green triangles. The green error bars now reflect both the precision and accuracy of the $W_{10}(\mathrm{H} \alpha)$ measurements and are described well by the sinusoidal fit.

\section{Discussion}

The observed $0.16 \mathrm{~d}$ period might be caused by the rotational modulation of one or more $\operatorname{spot}(s)$ on the stellar surface. Adopting the stellar radius of $1.4 \pm 0.25 R_{\odot}$ and $v \sin i_{\star}=$ $82.5 \pm 2.9 \mathrm{~km} \mathrm{~s}^{-1}$ from Table 1 , the inclination of the stellar rotation axis is $i_{\star}=11^{\circ} \pm 2^{\circ}$. We thus see the star almost pole-on. This could explain the weak correlation between the BVS and $R V_{\star}$ (Sect. 4.3). The disk of HD 135344B is also found to be almost face-on with inclination measurements of $\lesssim 20^{\circ}$ (Grady 
et al. 2009) and $11^{\circ} \pm 2^{\circ}$ by Dent et al. (2005), i.e., the orientation of the stellar rotation axis, based on the assumption that the $0.16 \mathrm{~d} R V_{\star}$ period represents the stellar rotation period, is almost perpendicular to the mid-plane of the outer disk. This supports the hypothesis that the $0.16 \mathrm{~d}$ period is caused by photospheric effects.

Another approach to verifying the origin of the $0.16 \mathrm{~d}$ period is to apply a Keplerian fit to the data. Under the assumption of a circular orbit (eccentricity is set to 0 ) and a period of $0.16 \mathrm{~d}$, the semi-major axis is $7 \times 10^{-3} \mathrm{AU}$, which corresponds to $1.5 R_{\odot}$. For HD 135344B, we found the stellar radius to be $1.4 \pm 0.25 R_{\odot}$ (Table 1), i.e, the semi-major axis to be in the range of the stellar radius. This shows that the companion hypothesis is implausible, thus favors the photospheric interpretation. Thus, the rotational period of $0.16 \mathrm{~d}$ or $3.9 \mathrm{~h}$ makes HD $135344 \mathrm{~B}$ an extremely rapid rotator.

\subsection{HD 135344B is rotating close to break-up velocity}

The break-up velocity $v_{\mathrm{c}}=\sqrt{G M_{\star} / R_{\star}}$ of the star, where $G$ is the gravitational constant, has a value of $480 \pm 60 \mathrm{~km} \mathrm{~s}^{-1}$. Using the value of $i_{\star}=11^{\circ} \pm 2^{\circ}$ derived from the stellar parameters leads to a true rotational velocity of $432 \pm 81 \mathrm{~km} \mathrm{~s}^{-1}$. Therefore, HD $135344 \mathrm{~B}$ is rotating at or close to the break-up velocity at its equator.

Aufdenberg et al. (2006) interferometrically measured the polar and equatorial radius of Vega, which rotates at $91 \%$ of its break-up velocity. They measured a difference of $20 \%$ between the equatorial and polar radius and found the temperature gradient to be $2250 \mathrm{~K}$. For HD 135344B, similar effects are expected. Because the star is seen almost pole-on, we might overestimate its luminosity, thus its mass and temperature. The angular stellar diameter of HD 135344B is about $100 \mu$ as and not resolvable by current stellar interferometric facilities, preventing a direct confirmation and determination of its true stellar parameters.

The continuous accretion of circumstellar material onto the star could have caused the spin-up of the stellar rotation up to $v_{\mathrm{c}}$. HD 135344B has only a very weak magnetic field (Hubrig et al. 2009). Therefore, the star is probably decoupled from its disk, which prevents the star from magnetic braking and losing spin. In contrast, for the majority of TTSs only moderate $v \sin i_{\star}$ values are observed, which indicates that a disk-braking mechanism operates during the accretion phase (e.g. Weise et al. 2010).

\subsection{Possible origins of the observed periodicity}

\subsubsection{The von Zeipel effect}

Because of its rapid rotation, the stellar atmosphere of the star should be oblate because of the centrifugal force. This causes a redistribution of the flux, which is proportional to the local surface gravity (von Zeipel 1924), i.e., the equatorial zones become darker at a lower $T_{\text {eff }}$ than the polar zones. This phenomenon is known as gravity-darkening and is symmetric with respect to the equator. To produce a periodic $R V_{\star}$ signal, i.e. mimic a stellar spot, the flux and temperature gradient has to be off-center from the rotational axis to produce a Doppler shift, i.e. the von Zeipel effect cannot account for the observed periodicity of the spectra. Smith \& Worley (1974) and Claret (2000) showed that for differentially rotating radiative stellar atmospheres, the flux over the stellar surface varies more strongly than the surface gravity, which could produce asymmetric flux distributions.

\subsubsection{Stellar spots}

We computed different spot configurations (temperature differences of the spot with respect to the photosphere $\Delta T_{\text {spot }}$, filling factor $f_{\text {spot }}$, and latitude of the spot $\Theta$ ) by taking the determined stellar parameters into account.

The effects of star spots on photometry and $R V_{\star}$ were numerically derived with a relatively simple model. A sphere with radius $R$, unit surface brightness $I$, and one or more round spots with spot filling factor $f_{\text {spot }}$ at a specific latitude and longitude was projected onto a $2 \mathrm{D}$ Cartesian grid with $200 \times 200$ pixels. The relative spot brightness at the considered wavelength was calculated from the ratio of effective photospheric temperature to spot temperature, assuming black-body radiation. To account for opacity effects on the stellar surface, a linear limb darkening law with coefficients adopted from Claret \& Hauschildt (2003) was applied. The inclination of the rotation axis can be arbitrarily chosen. Solid-body rotation was assumed, but differential rotation was not considered. Each cell of the star image was thus assigned a brightness and a radial velocity value. The surface brightness distribution at each phase of the stellar rotation (in steps of $1 \mathrm{deg}$ ) was then integrated to obtain the total brightness and the position of the photo center (1st moment). The first moment of the radial velocity distribution, weighted with the surface brightness distribution, was adopted as a proxy for the the mean radial velocity. Absorption line shapes were not taken into account.

The two extreme scenarios that can explain the $R V_{\star}$ variations are: 1.) dark spot, $\Delta T_{\text {spot }}=-1350 \mathrm{~K}, \Theta=40^{\circ}, f_{\text {spot }}=5 \%$, and 2.) bright spot, $\Delta T_{\text {spot }}=2000 \mathrm{~K}, \Theta=85^{\circ}, f_{\text {spot }}=56 \%$. The large covering fraction of the latter model may more closely resemble the von Zeipel effect than traditional star spots. There is still no available photometric monitoring data for HD 135344B that would set additional constraints on the possible spot configurations.

We conclude that both explanations of the origins of the observed $R V_{\star}$ variations, such as stellar spots or the flux and temperature gradient between the pole and equator, can adequately explain the data.

Owing the accretion process, the $W_{10}(\mathrm{H} \alpha)$ variations may trace a hot spot from an accretion funnel flow that has its origin at the inner edge of the inner disk. A periodic variation in $5.77 \mathrm{~d}$ is found for $W_{10}(\mathrm{H} \alpha)$ (Sect. 4.4). This period corresponds to a Keplerian radius of $\approx 0.07 \mathrm{AU}$, which is close to the inner edge of the disk estimated to $0.05 \mathrm{AU}$ by Fedele et al. (2008), given the uncertainty. A similar effect was observed for $E W(\mathrm{H} \alpha)$ variations for the TW Hya system by Setiawan et al. (2008).

Several studies have shown that the disk lifetimes of young stars are expected to be between $\sim 5$ and $\sim 10$ Myr (e.g. Haisch et al. 2001; Bouwman et al. 2006; Jayawardhana et al. 2006; Sicilia-Aguilar et al. 2006; Fedele et al. 2010). At this age, stars lose their inner dusty disk and accretion stops. This also seems to coincide with the dissipation of gas in the inner disk (Jayawardhana et al. 2006). In contrast, HD 135344B displays accretion signatures, an inner dusty disk with an inclination, which is much greater than that of the outer disk, as well as a gasrich inner environment of age $9 \pm 2 \mathrm{Myr}$. A possible explanation might be that HD 135344B loses parts of its outer atmosphere because of its rapid rotation at or close to its break-up velocity, and feeds the inner part of the disk with its own material, which is then accreted back onto the star.

A possible stellar or sub-stellar companion located in the gap of the disk suggested by literature (e.g. Grady et al. 2009) cannot be confirmed or ruled out with our measurements because our 
spectroscopic data set covers only five months in total and the uncertainties in the $R V_{\star}$ measurements are of about $300 \mathrm{~m} \mathrm{~s}^{-1}$.

\section{Conclusions}

We have presented the first direct measurement of the stellar rotational period of a Herbig star. The period was derived from $R V_{\star}$ measurements by cross-correlating optical high-resolution spectra with a synthetic spectrum. The rotational period of HD $135344 \mathrm{~B}$ was found to be $0.16 \mathrm{~d}$. In addition, we have determined new reliable stellar parameters for HD 135344B, independent of previous literature values, and demonstrated that it is possible to measure the $R V_{\star}$ of a Herbig star with a high $v \sin i_{\star}$ value. From the measured stellar parameters, we were able to estimate the stellar inclination to a value of $11^{\circ} \pm 2^{\circ}$. With these data, we concluded that HD 135344B is rotating at or close to its break-up velocity and feeding its stellar vicinity with gas and dust. This is the first piece of observational evidence that a young intermediate-mass star can rotate close to its break-up velocity.

The direct determination of the stellar rotational period by $R V_{\star}$ measurements may also be applicable to other Herbig $\mathrm{Ae} / \mathrm{Be}$ stars. A larger sample could therefore provide insight into the evolution of angular momentum, the presence of stardisk coupling mechanisms, and the presence of magnetic fields in these young intermediate-mass stars.

We also detected variations in the $W_{10}(\mathrm{H} \alpha)$ measurements with a period of $5.77 \mathrm{~d}$, corresponding to a Keplerian radius close to the inner edge of the inner disk of HD 135344B, which could be a hot spot caused by an accretion funnel flow.

Acknowledgements. We thank our colleagues T. Anguita, R. Lachaume, and S. Protopapa for carrying out the FEROS observations. We thank B. Acke for providing us an additional spectrum of HD 135344B. This research has made use of NASA's Astrophysics Data System Bibliographic Services.

\section{References}

Appenzeller, I. 1994, in The Nature and Evolutionary Status of Herbig Ae/Be Stars, ed. P. S. The, M. R. Perez, \& E. P. J. van den Heuvel, ASP Conf. Ser., 62,12

Aufdenberg, J. P., Mérand, A., Coudé du Foresto, V., et al. 2006, ApJ, 645, 664 Augereau, J. C., Lagrange, A. M., Mouillet, D., \& Ménard, F. 2001, A\&A, 365, 78

Barnes, III, T. G., Jeffery, E. J., Montemayor, T. J., \& Skillen, I. 2005, ApJS, 156, 227

Bevington, P. R., \& Robinson, D. K. 2003, Data reduction and error analysis for the physical sciences, ed. P. R. Bevington, \& D. K. Robinson

Boehm, T., \& Catala, C. 1995, A\&A, 301, 155

Bouwman, J., Lawson, W. A., Dominik, C., et al. 2006, ApJ, 653, L57

Brown, J. M., Blake, G. A., Dullemond, C. P., et al. 2007, ApJ, 664, L107

Castelli, F., \& Kurucz, R. L. 2004 [arXiv: astro-ph/0405087]

Catala, C., Felenbok, P., Czarny, J., Talavera, A., \& Boesgaard, A. M. 1986, ApJ, 308,791

Claret, A. 2000, A\&A, 359, 289

Claret, A., \& Hauschildt, P. H. 2003, A\&A, 412, 241

Coulson, I. M., \& Walther, D. M. 1995, MNRAS, 274, 977

Davis, R., Strom, K. M., \& Strom, S. E. 1983, AJ, 88, 1644

de Zeeuw, P. T., Hoogerwerf, R., de Bruijne, J. H. J., Brown, A. G. A., \& Blaauw, A. 1999, AJ, 117, 354

Dent, W. R. F., Greaves, J. S., \& Coulson, I. M. 2005, MNRAS, 359, 663

Desort, M., Lagrange, A., Galland, F., Udry, S., \& Mayor, M. 2007, A\&A, 473, 983

Doucet, C., Pantin, E., Lagage, P. O., \& Dullemond, C. P. 2006, A\&A, 460, 117 Dunkin, S. K., Barlow, M. J., \& Ryan, S. G. 1997, MNRAS, 286, 604

Fedele, D., van den Ancker, M. E., Acke, B., et al. 2008, A\&A, 491, 809

Fedele, D., van den Ancker, M. E., Henning, T., Jayawardhana, R., \& Oliveira,

J. M. 2010, A\&A, 510, A72

Finkenzeller, U. 1985, A\&A, 151, 340

\section{Appendix A: $R V_{\star}$ and $H \alpha$ measurements}

Table A.1. Measured $R V_{\star}, E W(\mathrm{H} \alpha), W_{10}(\mathrm{H} \alpha)$, and their corresponding uncertainties.

\begin{tabular}{|c|c|c|c|c|c|c|}
\hline $\begin{array}{l}\text { Time } \\
{[\mathrm{JD}-2400000 \mathrm{~d}]}\end{array}$ & $\begin{array}{c}R V_{\star} \\
{\left[\mathrm{m} \mathrm{s}^{-1}\right]}\end{array}$ & $\begin{array}{l}\sigma_{R V_{\star}} \\
{\left[\mathrm{m} \mathrm{s}^{-1}\right]}\end{array}$ & $\begin{array}{c}E W(\mathrm{H} \alpha) \\
{[\AA]}\end{array}$ & $\begin{array}{c}\sigma_{E W(\mathrm{H} \alpha)} \\
{[\AA]}\end{array}$ & $\begin{array}{c}W_{10}(\mathrm{H} \alpha) \\
{[\AA]}\end{array}$ & $\begin{array}{c}\sigma_{W(\mathrm{H} \alpha)} \\
{[\AA \AA]}\end{array}$ \\
\hline 55256.86690 & 2645 & 335 & -6.02 & 0.21 & 9.80 & 0.07 \\
\hline 55346.70410 & 1593 & 302 & -9.84 & 0.13 & 9.14 & 0.05 \\
\hline 55347.63521 & 1859 & 315 & -10.44 & 0.17 & 8.54 & 0.06 \\
\hline 55348.73788 & 2041 & 260 & -13.17 & 0.19 & 9.24 & 0.02 \\
\hline 55349.74833 & 2223 & 400 & -11.42 & 0.20 & 8.45 & 0.08 \\
\hline 55351.69950 & 2331 & 112 & -12.80 & 0.24 & 8.45 & 0.03 \\
\hline 55355.49994 & 2108 & 192 & -8.83 & 0.12 & 8.37 & 0.08 \\
\hline 55355.68805 & 2300 & 195 & -8.96 & 0.13 & 8.73 & 0.10 \\
\hline 55355.85300 & 1784 & 784 & -9.85 & 0.20 & 8.84 & 0.11 \\
\hline 55356.78750 & 1529 & 303 & -8.24 & 0.20 & 9.35 & 0.07 \\
\hline 55358.63746 & 3925 & 494 & -9.17 & 0.15 & 9.20 & 0.03 \\
\hline 55358.71724 & 2364 & 408 & -9.15 & 0.14 & 9.21 & 0.06 \\
\hline 55360.72711 & 2664 & 358 & -10.35 & 0.13 & 8.86 & 0.13 \\
\hline 55362.48448 & 2432 & 215 & -9.55 & 0.15 & 7.78 & 0.04 \\
\hline 55362.57222 & 1732 & 205 & -9.67 & 0.11 & 7.97 & 0.07 \\
\hline 55396.51686 & 3415 & 245 & -10.48 & 0.20 & 7.63 & 0.06 \\
\hline 55396.57497 & 2641 & 306 & -10.73 & 0.08 & 7.90 & 0.03 \\
\hline 55396.61357 & 2388 & 123 & -10.91 & 0.17 & 8.37 & 0.02 \\
\hline 55396.66325 & 3566 & 214 & -10.90 & 0.16 & 8.69 & 0.05 \\
\hline 55396.69535 & 2672 & 382 & -11.18 & 0.08 & 8.75 & 0.03 \\
\hline 55397.52834 & 1992 & 339 & -10.01 & 0.11 & 8.27 & 0.05 \\
\hline 55397.59554 & 2288 & 247 & -9.99 & 0.13 & 8.11 & 0.11 \\
\hline 55397.64898 & 2739 & 381 & -10.02 & 0.30 & 8.05 & 0.03 \\
\hline 55397.68781 & 2866 & 241 & -10.27 & 0.15 & 8.06 & 0.09 \\
\hline 55398.51102 & 1932 & 519 & -11.25 & 0.29 & 8.45 & 0.09 \\
\hline 55400.51882 & 2692 & 303 & -10.66 & 0.14 & 10.03 & 0.06 \\
\hline 55400.57368 & 2159 & 173 & -10.18 & 0.22 & 9.65 & 0.06 \\
\hline 55400.61784 & 2891 & 239 & -9.70 & 0.14 & 9.64 & 0.10 \\
\hline 55400.67505 & 2803 & 321 & -9.52 & 0.20 & 9.41 & 0.07 \\
\hline 55401.48617 & 3341 & 376 & -10.65 & 0.17 & 9.20 & 0.03 \\
\hline 55401.63979 & 3461 & 215 & -10.01 & 0.17 & 9.23 & 0.02 \\
\hline 55401.69736 & 1035 & 546 & -9.56 & 0.21 & 8.93 & 0.07 \\
\hline 55402.56980 & 2032 & 374 & -9.41 & 0.00 & 8.63 & 0.06 \\
\hline 55402.62734 & 3849 & 347 & -9.35 & 0.24 & 8.36 & 0.11 \\
\hline 55402.69271 & 1769 & 433 & -8.77 & 0.18 & 8.09 & 0.06 \\
\hline 55403.56511 & 2802 & 378 & -8.49 & 0.19 & 8.81 & 0.04 \\
\hline 55403.59827 & 2209 & 193 & -8.08 & 0.23 & 8.48 & 0.13 \\
\hline 55403.63910 & 2810 & 471 & -8.05 & 0.19 & 8.60 & 0.11 \\
\hline 55403.67692 & 1898 & 311 & -7.99 & 0.23 & 8.59 & 0.11 \\
\hline 55404.50509 & 3058 & 250 & -8.80 & 0.18 & 9.55 & 0.05 \\
\hline 55404.67289 & 2932 & 247 & -9.56 & 0.18 & 9.61 & 0.06 \\
\hline 55405.63121 & 2431 & 197 & -12.65 & 0.16 & 9.74 & 0.07 \\
\hline 55405.68095 & 2686 & 313 & -12.62 & 0.19 & 9.55 & 0.04 \\
\hline 55407.58835 & 3106 & 371 & -10.67 & 0.16 & 8.52 & 0.02 \\
\hline
\end{tabular}

Finkenzeller, U., \& Mundt, R. 1984, A\&AS, 55, 109

Garcia Lopez, R., Natta, A., Testi, L., \& Habart, E. 2006, A\&A, 459, 837

Grady, C. A., Schneider, G., Sitko, M. L., et al. 2009, ApJ, 699, 1822

Gray, R. O., \& Corbally, C. J. 1994, AJ, 107, 742

Haisch, Jr., K. E., Lada, E. A., \& Lada, C. J. 2001, ApJ, 553, L153

Herbig, G. H. 1960, ApJS, 4, 337

Hubrig, S., Stelzer, B., Schöller, M., et al. 2009, A\&A, 502, 283

Hubrig, S., Mikulášek, Z., González, J. F., et al. 2011, A\&A, 525, L4

Jayawardhana, R., Coffey, J., Scholz, A., Brandeker, A., \& van Kerkwijk, M. H. 2006, ApJ, 648, 1206

Jeffery, E. J., Barnes, III, T. G., Skillen, I., \& Montemayor, T. J. 2007, ApJS, 171,512

Kaufer, A., Stahl, O., Tubbesing, S., et al. 1999, The Messenger, 95, 8

Manoj, P., Bhatt, H. C., Maheswar, G., \& Muneer, S. 2006, ApJ, 653, 657

Mason, B. D., Wycoff, G. L., Hartkopf, W. I., Douglass, G. G., \& Worley, C. E. 2001, AJ, 122, 3466

Natta, A., Testi, L., Muzerolle, J., et al. 2004, A\&A, 424, 603

Pontoppidan, K. M., Blake, G. A., van Dishoeck, E. F., et al. 2008, ApJ, 684, 1323 
A. Müller et al.: HD 135344B: a young star has reached its rotational limit

Pott, J., Perrin, M. D., Furlan, E., et al. 2010, ApJ, 710, 265

Povich, M. S., Giampapa, M. S., Valenti, J. A., et al. 2001, AJ, 121, 1136

Praderie, F., Catala, C., Simon, T., \& Boesgaard, A. M. 1986, ApJ, 303, 311

Preibisch, T., \& Mamajek, E. 2008, The Nearest OB Association: ScorpiusCentaurus (Sco OB2), ed. B. Reipurth, 235

Queloz, D., Henry, G. W., Sivan, J. P., et al. 2001, A\&A, 379, 279

Setiawan, J., Henning, T., Launhardt, R., et al. 2008, Nature, 451, 38

Sicilia-Aguilar, A., Hartmann, L., Calvet, N., et al. 2006, ApJ, 638, 897

Siess, L., Dufour, E., \& Forestini, M. 2000, A\&A, 358, 593

Smith, R. C., \& Worley, R. 1974, MNRAS, 167, 199
Thi, W. F., van Dishoeck, E. F., Blake, G. A., et al. 2001, ApJ, 561, 1074 van Boekel, R., Min, M., Waters, L. B. F. M., et al. 2005, A\&A, 437, 189 van der Plas, G., van den Ancker, M. E., Fedele, D., et al. 2008, A\&A, 485, 487

Verhoeff, A. P., Waters, L. B. F. M., Veerman, H., et al. 2010, A\&A, submitted von Zeipel, H. 1924, MNRAS, 84, 665

Waters, L. B. F. M., \& Waelkens, C. 1998, ARA\&A, 36, 233

Weise, P., Launhardt, R., Setiawan, J., \& Henning, T. 2010, A\&A, 517, A88

White, R. J., \& Basri, G. 2003, ApJ, 582, 1109

Zechmeister, M., \& Kürster, M. 2009, A\&A, 496, 577 